\title{
Strategies and Tools for ATLAS Online Monitoring
}

W. Vandelli, P. Adragna, D. Burckhart, M. Bosman, M. Caprini, A. Corso-Radu, M. J. Costa, M. Della Pietra, J. Von Der Schmitt, A. Dotti, I. Eschrich, M. L. Ferrer, R. Ferrari, G. Gaudio, H. Hadavand, S. J. Hillier, M. Hauschild, B. Kehoe, S. Kolos, K. Kordas, R. Mcpherson, M. Mineev, C. Padilla, T. Pauly, I. Riu, C. Roda, D. Salvatore, I. Scholtes, S. Sushkov, H. Wilkens, and P. F. Zema

\begin{abstract}
ATLAS is one of the four experiments under construction along the Large Hadron Collider (LHC) ring at CERN. The LHC will produce interactions at a center-of-mass energy equal to $\sqrt{\mathrm{s}}=14 \mathrm{TeV}$ with a frequency of $40 \mathrm{MHz}$. The detector consists of more than 140 million electronic channels. The challenging experimental environment and the extreme detector complexity impose the necessity of a common, scalable, distributed monitoring framework, which can be tuned for optimal use by different ATLAS sub-detectors at the various levels of the ATLAS data flow. This paper presents the architecture of this monitoring software framework and describes its current implementation, which has already been used at the ATLAS beam test activity in 2004. Preliminary performance results, obtained on a computer cluster consisting of 700 nodes, will also be presented, showing that the performance of the current implementation is within the range of the final ATLAS requirements.
\end{abstract}

Index Terms-Data acquisition, HEP, monitoring, software performance.

Manuscript received November 28, 2006; revised February 12, 2007.

W. Vandelli is with Università di Pavia and INFN Sezione di Pavia, 27100 Pavia, Italy.

P. Adragna is with Queen Mary, University of London, London E1 4NS, U.K., on leave from Università degli Studi di Siena e INFN Sezione di Pisa, 56127 Pisa, Italy.

D. Burckhart, M. J. Costa, M. Hauschild, C. Padilla, T. Pauly, and H. Wilkens are with CERN, 1211 Geneva 23, Switzerland.

M. Bosman and S. Sushkov are with IFAE, Institut de Fisica de Altes Energies, $08193 \mathrm{UAB} /$ Barcelona, Spain.

M. Caprini is with National Institute for Physics and Nuclear Engineering, 76900 Bucharest, Romania.

A. Corso-Radu and I. Eschrich are with University of California, Irvine, Irvine, CA 92664 USA.

M. Della Pietra is with INFN Sezione di Napoli, 80125 Napoli, Italy.

J. Von Der Schmitt is with MPI for Physics, 80805 Munich, Germany.

A. Dotti and C. Roda are with Università di Pisa and INFN Sezione di Pisa, 56127 Pisa, Italy.

M. L. Ferrer and K. Kordas are with INFN Laboratori Nazionali di Frascati, 00044 Frascati, Italy.

R. Ferrari and G. Gaudio are with INFN Sezione di Pavia, 27100 Pavia, Italy.

H. Hadavand and B. Kehoe are with Southern Methodist University, Dallas, TX 75222 USA.

S. J. Hillier is with University of Birmingham, Birmingham B5 4AD, U.K.

S. Kolos is with University of California, Irvine, Irvine, CA 92664 USA, on leave from PNPI, Gatchina, Russia.

R. Mcpherson is with University of Victoria, Victoria BC V8W 2Y2, Canada.

M. Mineev is with JINR, 141980, Dubna, Moscow Region, Russia.

I. Riu is with Université de Geneve, 1211 Geneva 4, Switzerland.

D. Salvatore is with Università della Calabria and INFN Sezione di Cosenza, 87036 Arcavacata di Rende (Cosenza), Italy.

I. Scholtes is with the University of Trier, 54296 Trier, Germany.

P. F. Zema is with CERN, 1211 Geneva 23, Switzerland, on leave from Università della Calabria and INFN Sezione di Cosenza, 87036 Arcavacata di Rende (Cosenza), Italy.

Color versions of one or more of the figures in this paper are available online at http://ieeexplore.ieee.org.

Digital Object Identifier 10.1109/TNS.2007.894368

\section{INTRODUCTION}

A TLAS [1] is one of the four experiments being installed at the Large Hadron Collider (LHC). The experiment includes several challenging detection technologies and is supported by a large, distributed trigger and data acquisition system (TDAQ). LHC will provide collisions at a center-of-mass energy of $14 \mathrm{TeV}$ with a frequency of $40 \mathrm{MHz}$. The output of the ATLAS first level trigger will be less than $75 \mathrm{kHz}$. This frequency will be further reduced by the higher trigger levels and finally some hundreds of events will be selected and stored every second. The whole ATLAS detector consists of about 140 million electronic channels and the expected average event size is about 1-2 MB.

Considering the huge number of channels and the high event rate, a monitoring system is an essential tool to assess the status of the hardware and the quality of the data while they are being acquired. Such a monitoring system should cope with the challenging experimental conditions providing a flexible, scalable and tunable framework, in order to be useful for the different ATLAS sub-detectors and sub-systems.

\section{ATLAS TDAQ}

In ATLAS there are several levels of data flow [2]. Data are acquired by the front-end electronics (FE), located next to the detectors, and are collected, step by step, until the full event is assembled (see Fig. 1).

The first-level trigger electronics (LVL1) is devoted to the first selection level; if an event is accepted, all the FE boards send the data to the Read Out Drivers (RODs), which are detector-specific custom modules. Each ROD is point-to-point connected to one Read Out Buffer (ROB), which is a PCI custom board, to which it sends detector-specific data.

The event is then assigned to one processing node of the second-level trigger (LVL2) farms, which collects from the Read Out Systems (ROSs) the data fragments belonging to the detector regions selected by the LVL1 and starts its filtering algorithms. Accepted events are assigned to a Sub-Farm Input (SFI), which collects all the data fragments from the ROSs and assembles the complete event. This is also called Event Building (EB) stage.

The last filtering stage is the Event Filter (EF), the second component, together with the LVL2, of the High-Level Trigger (HLT) sub-system. Built events are sent to EF farm processing nodes, in which a Processing Task (PT) completely reconstructs and analyzes the data with high-precision algorithms taken from the ATLAS offline analysis framework (Athena). Events accepted by the EF are passed to the Sub-Farm Output (SFO) for transmission to mass storage. 


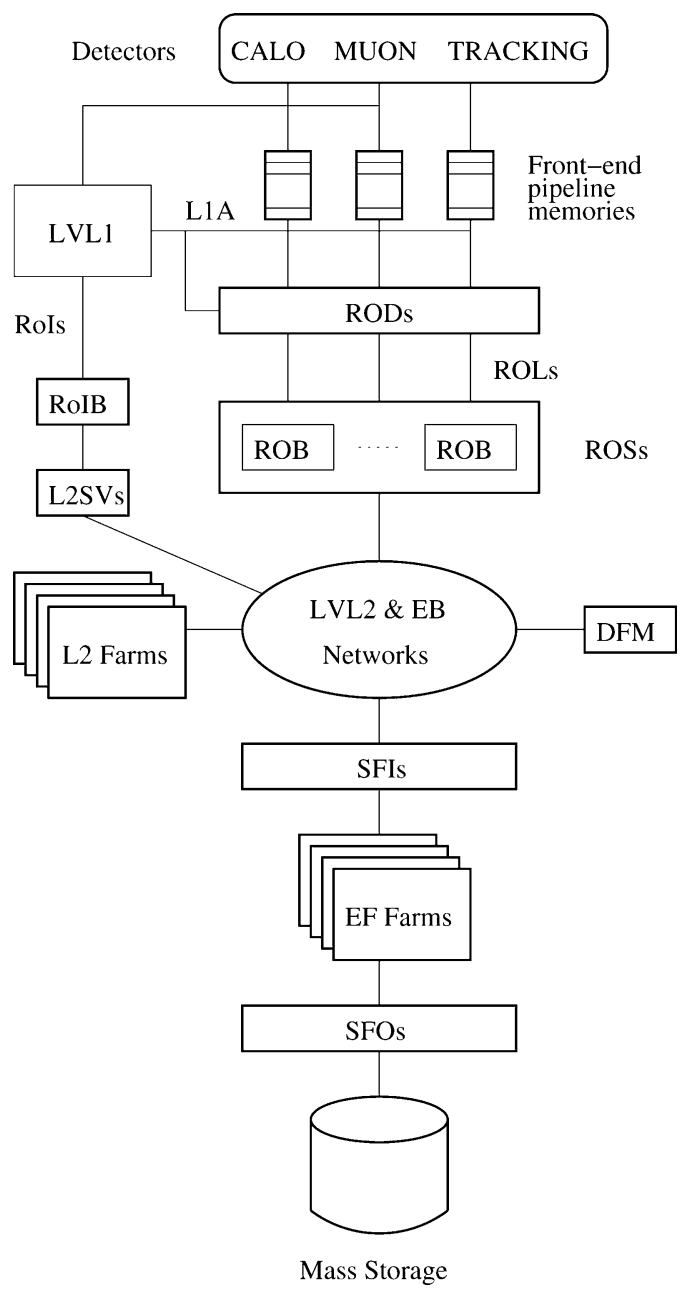

Fig. 1. ATLAS TDAQ layout.

The ATLAS TDAQ infrastructure is a large, distributed environment, including thousands of computing nodes and custom modules.

\section{MONITORING FRAMEWORK}

In order to verify the good quality of the data sent to the permanent storage, the whole triggering system, the DAQ system and the ATLAS sub-detectors should be constantly monitored in terms of functionality and results. The main requirements for the ATLAS monitoring framework are as follows:

- it should be able to cope with the detector complexity, being scalable and tunable to comply with all the different needs;

- it should support dynamic changes of the monitoring parameters;

- it should provide flexible and configurable Graphical User Interfaces (GUIs) to show the status of the monitored items;

- it should be capable to perform automatic checking of the monitoring results, including generation and distribution of alarms.

To understand the complexity of the monitoring framework mandate, it must be considered that more than 3000 sources of monitoring information and up to 300 possible event sampling points are foreseen in the final ATLAS environment. The monitoring framework will have to route, transport and manage different data types (e.g., event fragments, scalers, histograms) with different rates and loads depending on the sub-detector requirements [3]. Moreover the foreseen monitoring data produced during each run will be in the $O(10 \mathrm{~GB})$.

To fulfill this mandate, the ATLAS monitoring system is organized as a distributed framework and includes several applications, ranging from low-level information-sharing components up to high-level graphical interfaces. This separation permits isolation of the problems and optimisation of each application for the specific needs. The main components of the framework are shown in Fig. 2, while the diagram in Fig. 3 presents software architecture and component dependencies.

The main programming languages used in the development of the monitoring components are $\mathrm{C}++$ and Java. Usually $\mathrm{C}++$ is used where performance are fundamental: applications related to data handling and monitoring services. Java is instead used for graphical applications because of its wide graphical libraries.

Applications belonging to the monitoring framework are included in the global ATLAS TDAQ framework. Hence they exploit the same development and deployment tools: in particular CMT [4] is used for the management of the building and development environments, while software releases can be installed through ad-hoc shell scripts via AFS as well as exploiting RPM [5] packages.

However, the monitoring framework has also dependencies on packages and tools not included in the ATLAS TDAQ releases. The main dependencies are on ROOT [6] and CORBA [7], whose aims will be described in the following paragraphs. However, since CORBA is a well defined standard, our software, complying with the standard, is shielded by the actual implementation of the CORBA library. As far as ROOT is concerned, although a standard interface is not available as for CORBA, it should be stressed that ROOT is widely used in the high energy physics community. Actually, one of main aims of the ROOT developers is to keep new releases as back-compatible as possible.

\section{ONLINE MONITORING SERVICES}

A fundamental feature provided by the monitoring framework is the routing of many sorts of data produced by the TDAQ components. These data may include simple parameters as well as more complex information, like histograms or event fragments. The online monitoring services [8], Information Service (IS), Online Histogramming Service (OHS), Event Monitoring Service (Emon) and Message Reporting System (MRS), are devoted to this task. They provide different information-sharing channels, abstracting the underlying complexity of the distributed environment and adopting network and CPU load minimisation algorithms. Online monitoring services are independent of the main data-flow stream and transport data and requests between sources and destinations. Moreover, they give the possibility to perform the operational monitoring, namely the collection of many functional parameters, published 


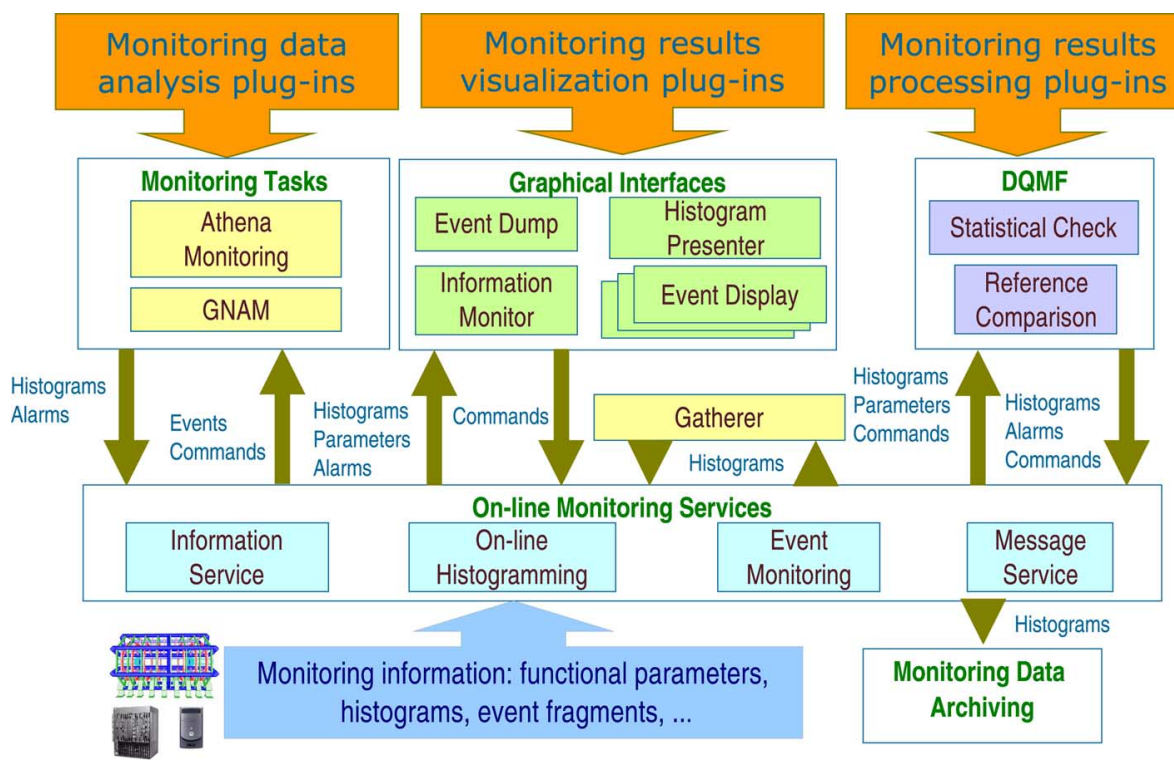

Fig. 2. Global view of the ATLAS online monitoring framework. Single components and their interactions are discussed in the text.

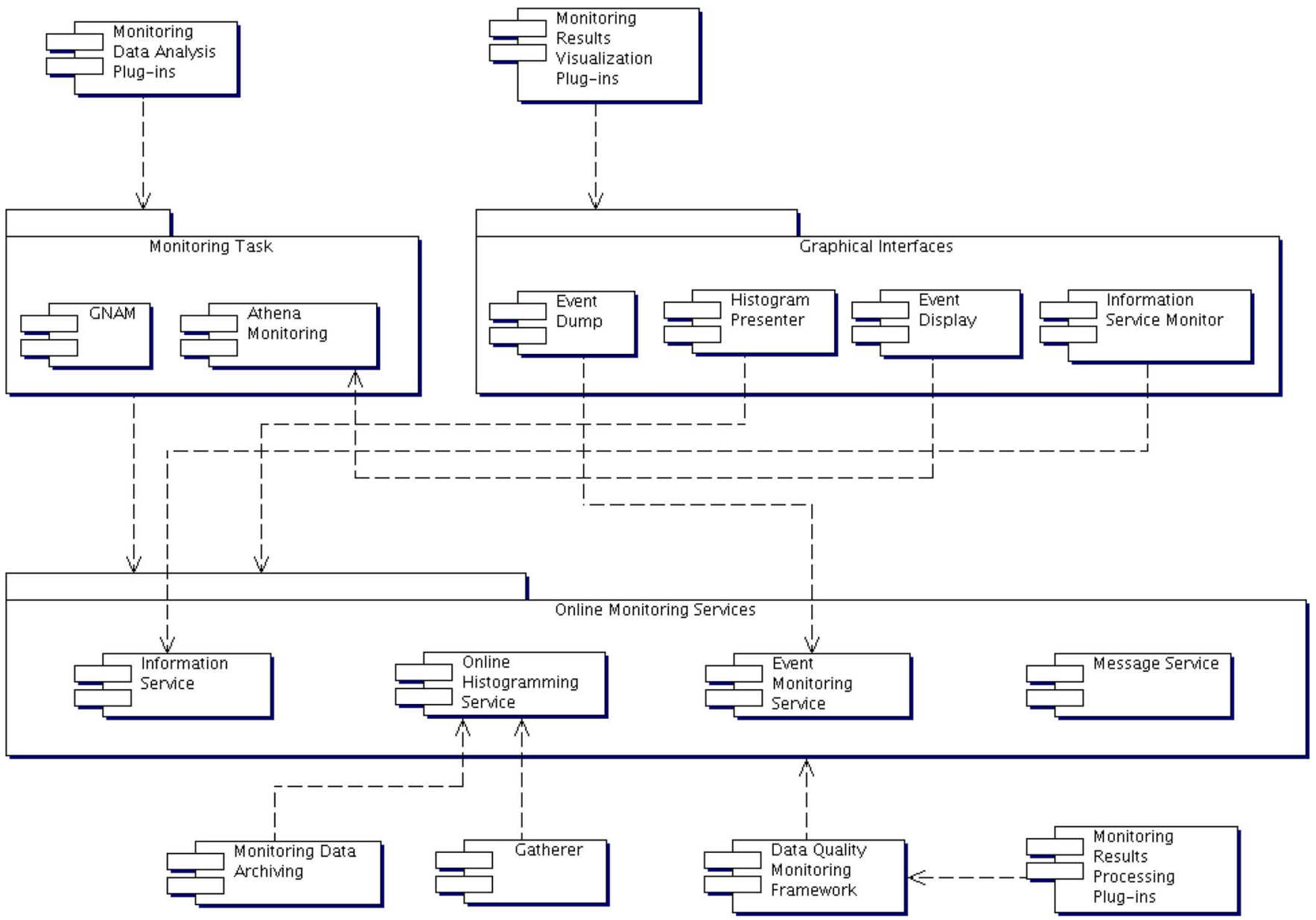

Fig. 3. Architecture of the ATLAS monitoring framework components. UML component diagram.

by hardware and software components, like busy statuses or data rates, and the production of time-trend plots.

The Inter Process Communication (IPC) [9] is a basic communication service, which is common for all the ATLAS online services. It defines a high-level API for the distributed object implementation and for the remote object location. IPC itself is built on top of CORBA, which provides the actual inter-object communication. The most important features of 
CORBA, inherited by the ATLAS online framework, are: object oriented communication, interoperability between different programming languages and object location transparency.

\section{A. Information Service (IS)}

The IS allows sharing of simple variables as well as user-defined data. A user can define the structure of his specific information in XML. Then, he can produce $\mathrm{C}++$ or Java information-handling classes using the generator application provided by the IS. These classes provide a description of user information and their instances can be shared between applications.

The IS supports three main types of interactions: information providers can create, update or delete information, while information readers can get the value of the information; moreover information receivers can subscribe to the repository to be notified about changes. In addition, through the IS any application is able to send commands to any of the running providers. This is useful to control the IS information flow: for example an application may ask a particular provider to increase the frequency of information updates or to republish a piece of information.

\section{B. Online Histogramming Service (OHS)}

The OHS is based on the IS and extends its functionalities to handle histogram objects, in particular raw and ROOT histograms. Raw histograms are simple data structures which describe a histogram through variables and arrays. In particular the histogram content is held in a data array.

The OHS is not responsible for booking, filling and presenting the histograms.

\section{Event Monitoring Service (Emon)}

Emon provides a framework to enable event sampling and distribution. User programs may request event fragments with selected properties, such as trigger or sub-detector type, from a specific sampling point. Since an event is transported as a sequence of bytes, Emon is neutral to the event format and can handle events coming from any level of the data flow. Indeed event samplers have been implement in all the components along the data path, from the ROD level up to the SFO level.

In order to minimise the load on the sampling application, requesting programs with the same selection criteria are arranged in a tree. Hence the sampling application forwards the events only to the first requester in any tree. The distribution of the data along the tree is done transparently to the users.

\section{Message Reporting System (MRS)}

MRS transports messages among TDAQ applications. Messages may be used to report debug information, warnings or error conditions. MRS allows association of qualifiers and parameters to each message. Message receivers can moreover subscribe to be notified about incoming messages, also using filtering criteria.

\section{MONitORing TASKS}

The possibility to analyse sampled events, producing histograms or other results, called data monitoring, is essential to assess the status of the sub-detectors and the functionality of
HLT and DAQ sub-systems. In the ATLAS monitoring framework, Monitoring Tasks supply this possibility, exploiting the online services to collect data and to make the results available. Indeed, even if data monitoring is directly performed inside the LVL2 and EF processing tasks, this will not be sufficient to ensure a complete control and cross-validation of the detector and TDAQ statuses. Hence, the Monitoring Tasks give the possibility to perform the data monitoring at any level of the data flow, taking advantage, for example, of the larger statistics available before the filtering stages, including the possibility to validate the HLT algorithms.

\section{A. Athena Monitoring}

The EF is a natural place to perform some monitoring activities: indeed events are completely reconstructed, leading to the possibility to re-use the information, to monitor high-level physics quantities and to perform cross-detector checks, without requiring additional $\mathrm{CPU}$ power.

Since high-level physics monitoring at different levels of the data flow is fundamental to check the filtering systems, the PT input-output system has been modified to transparently work with different data sources, also outside the EF framework. This led to Athena Monitoring [10], namely the possibility to exploit the offline algorithms on event fragments coming from any data flow level.

\section{B. Gatherer}

Inside the LVL2 and EF farms, many processing nodes run in parallel the same algorithms; therefore, to obtain meaningful information, the histograms they produce have to be summed together. A specific configurable application, the Gatherer [11], will run in background, requesting from the OHS the histograms published by the processing tasks and, in turn, publishing summary histograms.

\section{GNAM}

GNAM [12] (GNAM is Not AtlMon) is a light-weight configurable framework optimised for detector functionality monitoring. Indeed, this monitoring issue usually does not require complex analysis algorithms, hence the use of a dedicated lightweight framework minimises the use of computing resources.

GNAM can be used to perform many sorts of jobs, thanks to a plug-in design that separates common actions from analysis algorithms, which are stored in dynamic libraries loaded at run-time. GNAM can moreover handle asynchronous commands coming through the OHS to modify histogram properties at run-time or to execute custom functions defined in the analysis libraries.

\section{GRAPHICAL INTERFACES}

A complete monitoring system should also provide flexible and configurable GUIs to allow a fast and user-friendly control of the status of the monitored items.

The ATLAS monitoring framework provides viewers for the online services: IS Monitor can show the content of IS servers, while OH Display permits browsing of the histograms in the OHS server, acting on them with all the ROOT graphic features. 


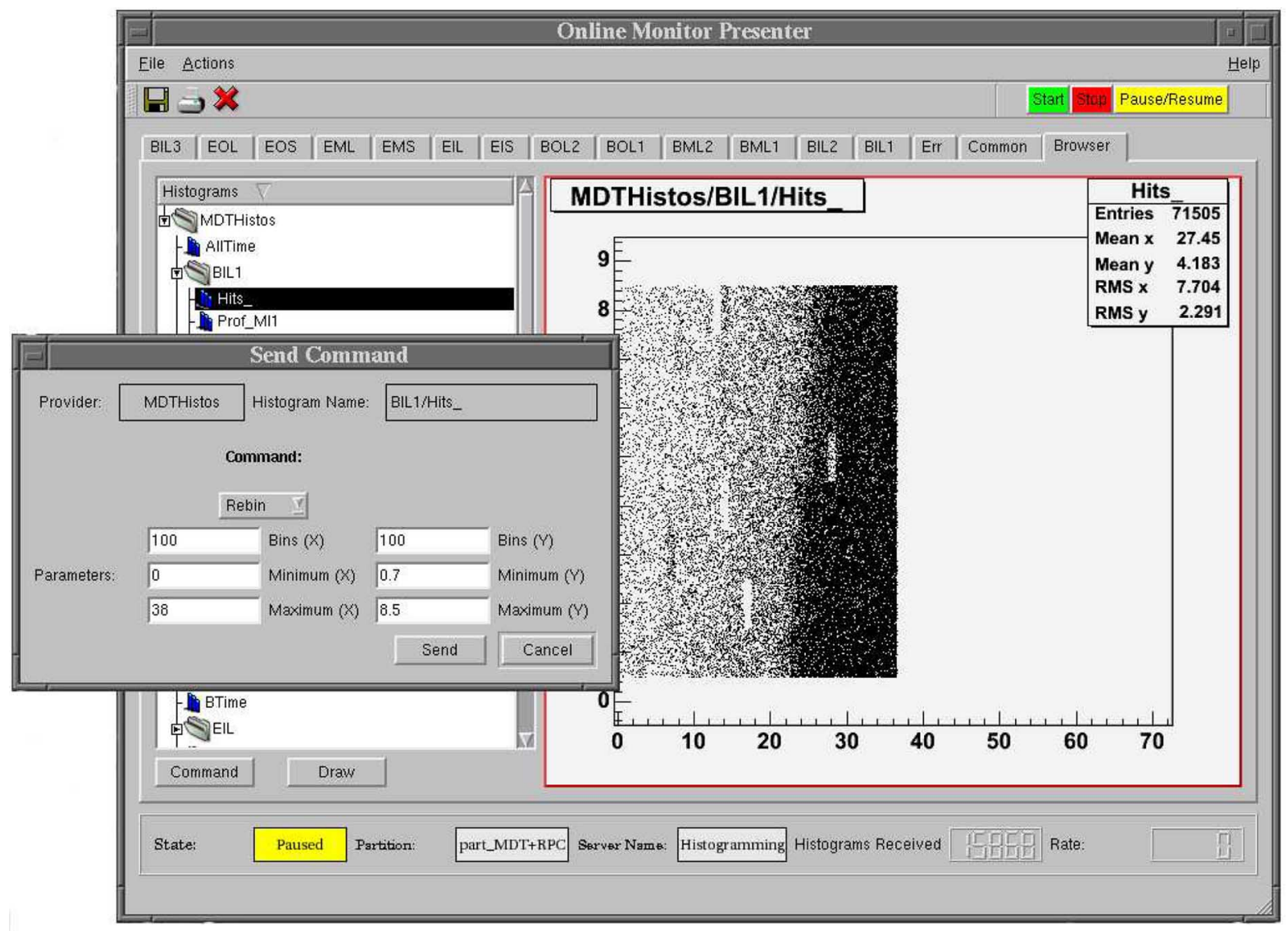

Fig. 4. OHP screen shot.

To assess the format of the data, Event Dump can sample events from data flow and show them in structured tables.

\section{A. Online Histogram Presenter (OHP)}

OHP [12] is a highly configurable histogram presenter based on ROOT and $\mathrm{Qt}^{\circledR}$ (Trolltech Inc.) [13]. It is structured as a multi-thread application, implementing a caching mechanism, which exploits the change notification feature of the OHS, preventing unchanged or out-of-scope histograms to be unnecessarily requested. Moreover OHP is based on a modular design which easily allows future extensions.

OHP can operate in two different modes: it can browse the OHS and/or show a configurable set of online or reference histograms in a series of tabs. Reference histograms can be shown superimposed or side-by-side to the corresponding online histograms. The two modes allow both the detector experts and the standard shifters to have all the needed functionalities within the same application. Furthermore ROOT context menus are available, enabling operations like fitting or zooming. Users can also send commands to the monitoring tasks using preconfigured buttons and panels. In Fig. 4, OHP in browser mode is shown with a superimposed command panel. On the left the content tree of the OHS is visible, while on top all the preconfigured tabs are present.

\section{FURTHER DEVELOPMENTS}

\section{A. Data Quality Monitoring Framework (DQMF)}

Due to the ATLAS complexity, a framework for reference histogram comparison, statistical checks and alarm generation is definitely needed. The DQMF mandate is to apply specific analysis algorithms to various types of monitoring data (e.g., histograms, messages, counters) according to a particular configuration, specified by the users. These algorithms will operate on the monitoring results from the monitoring tasks and will generate alarms when deviations are encountered. Moreover, a summary of the DQMF results will help experts to make the final data quality assessment for a given run. Hence DQMF has also to provide alarm routing mechanisms and GUIs to present analysis status and results.

The development of such a framework, after the collection of the user requirements, has just started [14] investigating software and experiences from other HEP experiments and implementing prototype functionalities in the existing tools.

\section{B. Monitoring Data Archiving (MDA)}

In ATLAS it is foreseen to have $O(10 \mathrm{~GB})$ of monitoring results per run. Most of this data have to be stored and kept available for a limited time, in order to be able to cross-check the offline analysis results with the online monitoring ones. Besides, such an archiving system will be useful to store reference histograms too. The monitoring system has therefore to provide 
common API and command-line tools to store, manage and retrieve the monitoring data.

The proposed architecture of MDA [15] includes a local histogram cache, which holds monitoring results after the end of run, and an offline archiver application, which stores the data in the mass storage and registers their location in a database, together with the conditions in which the data have been taken. This design avoids a delay in the end-of-run transition due to the time needed to store the monitoring results. Moreover, the use of a database enables queries for data corresponding to specific run conditions and requires access to the mass storage only for the data download itself.

\section{COMBINED TeST BEAM 2004}

ATLAS test beams always gave a strong motivation for the development of monitoring software. Particular is the case of the Combined Test Beam (CTB04) [16], carried out in summer 2004, in which a complete slice of the ATLAS detector was exposed to a particle beam. Indeed during the CTB04, the on-line services and the prototypes of Gatherer, GNAM and OHP were widely used, handling hundreds of histograms produced by different sub-detectors and becoming fundamental tools for both the shift crew and the detector experts.

\section{LARGE SCALE TeSt 2005}

During summer 2005, tests of functionality of the TDAQ system and of single selected components were carried out using up to 700 nodes of the LXBATCH cluster at CERN [17]. Most of the computers were equipped with a dual Pentium IV $2.8 \mathrm{GHz}$ processor and 0.5-2 GB of memory. The slowest network connection between those nodes was a Fast Ethernet.

IS, OHS and Emon components were comprehensively tested with positive results: no failures were observed and the scalability of the architecture has been proven. Test conditions, in terms of service load, have been chosen to be as close as possible to the final ones.

\section{A. Information Service}

The IS tests were carried out using 350 nodes, with 1 to 10 providers per node, publishing $250 \mathrm{~B}$ of data, and with 1 to 15 receivers reading all the available information. In ATLAS $O(10)$ receivers are foreseen, which however collect different data subsets.

The IS fulfilled the required performances, with publishing times in the order of $10 \mathrm{~ms}$ in the worst cases (Fig. 5). Indeed, the sudden increase of the time for the scenarios with 10 and 15 receivers is due to the saturation of the Fast Ethernet bandwidth, as shown in Fig. 6, whereas the proper ATLAS infrastructure will exploit a Gigabit network, hence providing a larger network bandwidth.

\section{B. Online Histogramming Service}

The OHS was tested using 650 nodes with one provider per node publishing one ROOT histogram every $10 \mathrm{~s}$, in the case

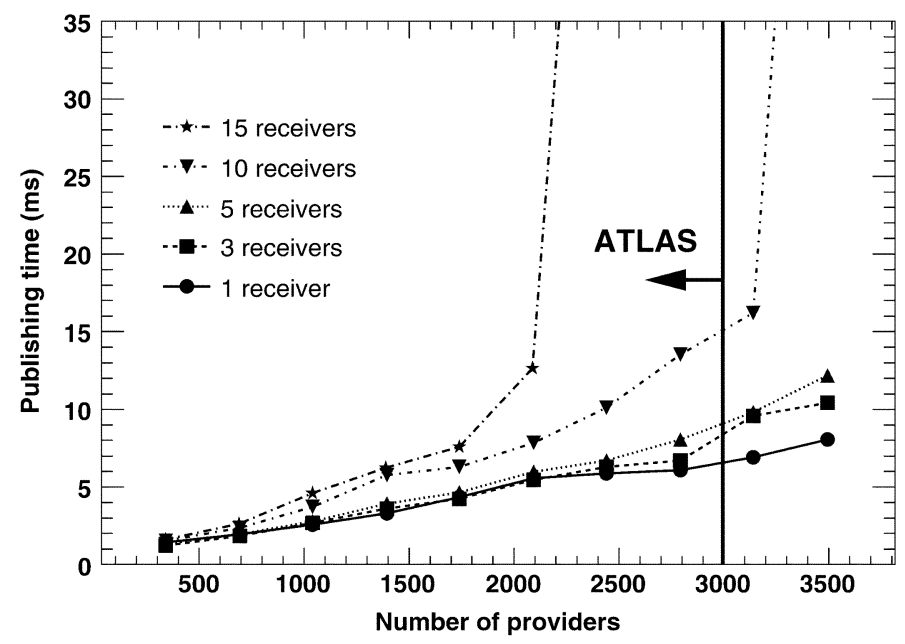

Fig. 5. Average IS publishing time as a function of the numbers of providers and receivers. The ATLAS working point should lie on the left of the shaded area.

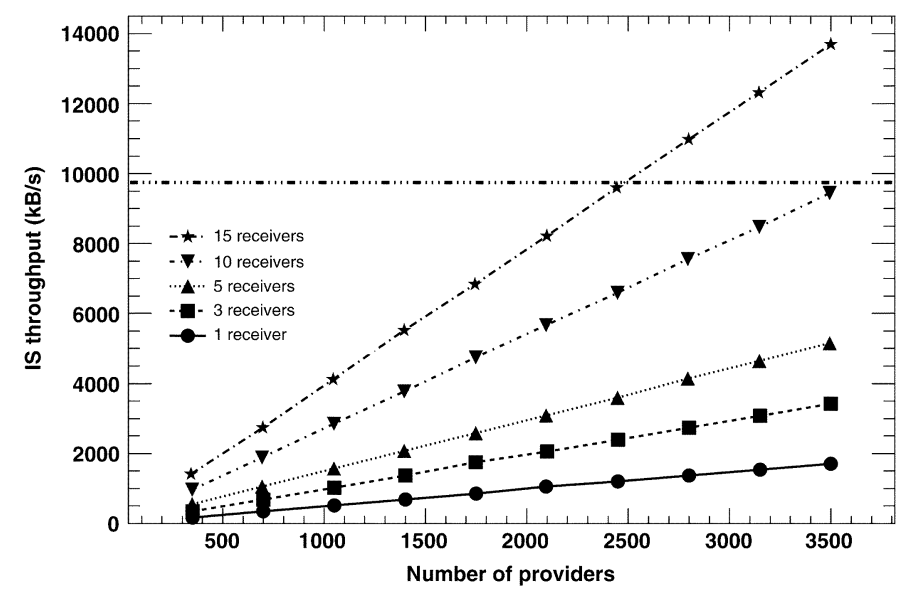

Fig. 6. IS throughput as a function of the numbers of providers and receivers. The horizontal line is the maximum Fast Ethernet bandwidth, on real networks the actual available bandwidth is always less due to protocol overhead and network latency.

of no receivers, or every $30 \mathrm{~s}$ in the case of two receivers. Receivers were collecting all the published histograms. Tests were performed using different histogram sizes, ranging from a few kilobytes up to about one megabyte. Indeed, for each bin in a ROOT histogram, the needed space includes 2 or $4 \mathrm{~B}$, depending on the data type (short or float bins), $8 \mathrm{~B}$ for the error information and $8 \mathrm{~B}$ since they were variable axis histograms.

The service performed well (Fig. 7), considering that, in the worst case, the OHS managed the publishing and the collection of roughly $650 \mathrm{MB}$ of data every $30 \mathrm{~s}$. However the OHS allows readers to subscribe only to be notified about changes, avoiding unnecessary histogram transportations and therefore reducing the system load.

As for the IS, in ATLAS it is expected to have many more than two histogram receivers, but subscribing for distinct histogram subsets. 


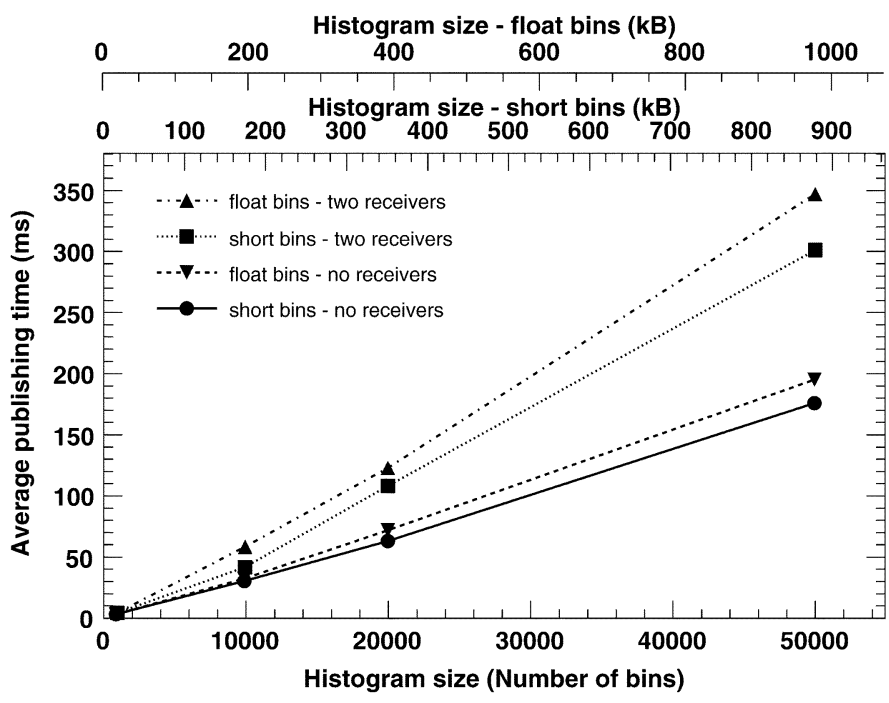

Fig. 7. Average OHS publishing time with 650 providers as a function of the numbers of histogram bins and receivers.

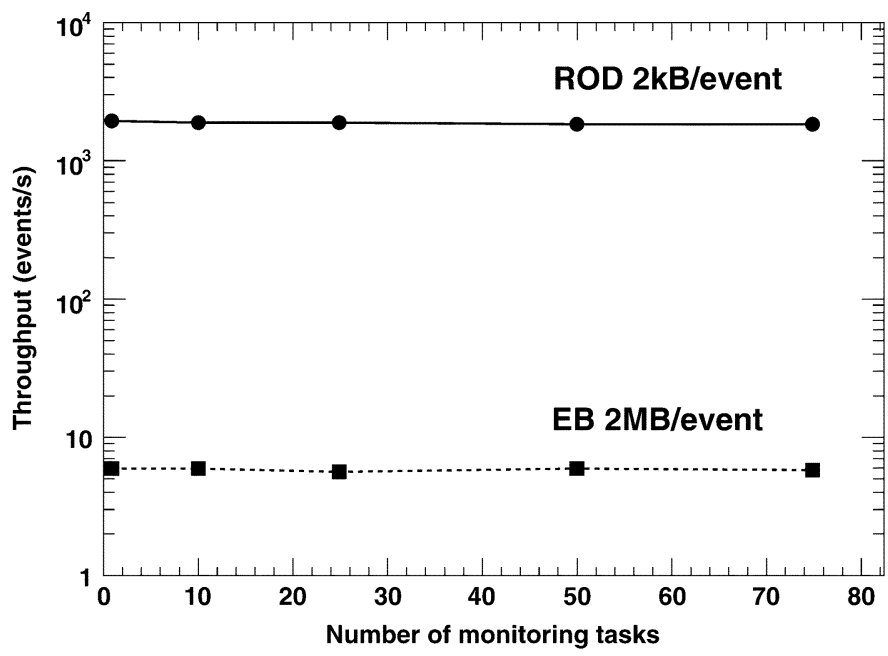

Fig. 8. Emon event distribution rate as a function of the number of sampling channels for two different scenarios.

\section{Event Monitoring Service}

The Emon performance were checked in two different scenarios: using the smallest ATLAS sub-fragment (ROD profile, $2 \mathrm{kB} / \mathrm{event}$ ) or the largest one (EB profile, $2 \mathrm{MB} / \mathrm{event}$ ). As can be seen in Fig. 8, Emon provides an almost constant event rate, regardless of the number of sampling channels, at least in the foreseen ATLAS working range. Even in the worst case, i.e., the EB profile, the provided rate of some hertz is large enough to fulfill the requirements of most of the monitoring scenarios.

Moreover during the test a negligible CPU utilization by the sampling thread was observed. This is quite important since the sampling thread will run on the same node where the data-flow applications handling the sampled data will run. Hence, the sampling application must need low computing resources in order to not affect the performances of the data-flow chain.

\section{CONCLUSION}

A first implementation of the monitoring framework for ATLAS is already available, from fundamental communication services to high-level analysis and graphical applications.

Existing monitoring components were used during the Combined Test Beam 2004, where they proved to be extensible and configurable enough to satisfy the different sub-detector needs and being a fundamental tool for the shift crew and the detector experts. Now, the same tools are used during the commissioning of sub-detectors to assess the hardware status. Moreover, preliminary tests on a large distributed environment suggest that, at least for the fundamental services, the actual implementation is suitable for ATLAS.

\section{REFERENCES}

[1] ATLAS Tech. Proposal, ATLAS Collaboration, (in CERN/ LHHCC/94-43, LHCC/P2). Geneva, Switzerland, 1994.

[2] ATLAS, High-Level Trigger, Data Acquisition and Controls, ATLAS Collaboration (in CERN/LHCC/2003-022). Geneva, Switzerland, 2003.

[3] Summary of the Monitoring Requirements [Online]. Available: https://edms.cern.ch/document/681546/1, ATLAS TDAQ Monitoring Working Group, Nov. 2005

[4] C. Arnault, "CMT: A software configuration management tool," in Proc. Computing in High Energy and Nuclear Physics, Padova, Italy, 2000, pp. 692-695.

[5] E. Bailey, Maximum RPM. Indianapolis, IN: Sams, 1997, ISBN 0-672-31105-4.

[6] R. Brun and F. Rademakers, "ROOT_-An object oriented data analysis framework," Nucl. Instrum. Methods Phys. Res. A, vol. A389, no. 1-2, pp. 81-86, Apr. 11, 1997.

[7] M. Henning and S. Vinoski, Advanced CORBA Programming with $C++$. Reading, MA: Addison-Wesley, 1999, ISBN 0-201-37927-9.

[8] S. Kolos, "Online monitoring software framework in the ATLAS experiment," in Proc. Computing in High Energy and Nuclear Physics, La Jolla, CA, 2003.

[9] A. Amorim, "Use of CORBA in the ATLAS prototype DAQ," IEEE Trans. Nucl. Sci., vol. 45, no. 4, pp. 1978-1982, Aug. 1998.

[10] M. Bosman, "Development and tests of the event filter for the ATLAS experiment," in Proc. 14th IEEE-NPSS Real Time Conf., Stockholm, Sweden, Jun. 2005

[11] P. Conde-Muino, "Portable gathering system for monitoring and online calibration at ATLAS," in Proc. Computing in High Energy and Nuclear Physics, Interlaken, Switzerland, 2004.

[12] P. Adragna, A. Dotti, R. Ferrari, C. Roda, W. Vandelli, and P. F. Zema, "GNAM: A low level monitoring program for the ATLAS experiment," IEEE Trans. Nucl. Sci., vol. 53, no. 3, pp. 1317-1322, Jun. 2006.

[13] J. Blanchette and M. Summerfield, C++ Programming with Qt 3. Englewood Cliffs, NJ: Prentice-Hall, 2004, ISBN 0-13-124072-2.

[14] A. Corso-Radu, H. Hadavand, M. Hauschild, B. Kehoe, and S. Kolos, Data Quality Monitoring Architectural Design, Aug. 2006 [Online]. Available: https://edms.cern.ch/document/770411/1.0

[15] P. F. Zema, Monitoring Data Archiving. Collection and Caching Service. Reference Histogram Management, Mar. 2006 [Online]. Available: https://edms.cern.ch/document/713107/1.1

[16] B. Di Girolamo, A. Dotti, V. Giangiobbe, P. Johansson, L. Pribyl, and M. Volpi, "Beamline instrumentation in the 2004 combined ATLAS testbeam," ATLAS Notes. Geneva, Switzerland, CERN, 2005, ATLTECH-PUB-2005-001.

[17] ATLAS DAQ/HLT Software Large Scale Functionality and Performance Tests July 2005 [Online]. Available: https://edms.cern.ch/file/ 685256/1/LSTReport.pdf, Nov. 2005, ATLAS HLT/DAQ developers and testers. 